Conclusions The SKB technique is safety and efficacy for the special ostial LAD lesions, long-term follow-up and large volume patients study is needed to verify the initial results.

\section{e0356 THE RESEARCH ON CALCIUM HOMEOSTASIS EXPRESSION AND GENE TRANSCRIPTION OF ATRIAL MYOCYTES IN PATIENTS WITH ATRIAL FIBRILLATION}

doi:10.1136/hrt.2010.208967.356

\begin{abstract}
${ }^{1}$ Mao Wei, ${ }^{2}$ Chen Junzhu, ${ }^{2}$ Yan Hui, ${ }^{2}$ Zheng Liangrong, ${ }^{2}$ Tao Qianmin, ${ }^{2}$ Ni Yiming. ${ }^{1}$ The First Affiliated Hospital of Zhejiang Traditional Chinese Medical University/ department of Cardiology; ${ }^{2}$ School of Medicine Zhejiang Universitydepartment of Cardiology
\end{abstract}

Objective Inquire into the molecular biological mechanism of the occurrence and maintenance of atrial fibrillation (AF) by researching adjustment and control of the $\mathrm{L}$ type the passage and muscles sarcoplasmic reticulum (SR) $\mathrm{Ca}^{2+}$ - the ATPase expression by main calcium ion $\left(\mathrm{Ca}^{2+}\right)$ of $\mathrm{AF}$ and the sinus rhythm.

Methods We took $200 \mathrm{mg}$ right auricle tissues and (or) left atrium tissues from each of the 63 patients undergoing cardiac surgery (including three groups: chronic AF, paroxysmal AF and sinus rhythm), extracted the protein and measured the density of overall sample protein, using Western-blot method to analyse the expression level of atria muscle L-type $\mathrm{Ca}^{2+}$ Passage and SR $\mathrm{Ca}^{2+}$ the ATPase. Isolated the total RNA of the artria muscle tissue by Trizol Method, measured the expression amount of atria muscle Ltype $\mathrm{Ca}^{2+}$ Passage and SR $\mathrm{Ca}^{2+}$-ATPase mRNA with RT-PCR method. Detailed clinical data were obtained before and after operation.

Results Firstly, left atrial diameter was obviously higher in patients with chronic AF or paroxysmal AF than in patients with sinus rhythm. Secondly, the patients with chronic AF have lower ratio of L-type $\mathrm{Ca}^{2+} / \mathrm{GAPDH}$ protein and SR $\mathrm{Ca}^{2+}$-ATPase/GAPDH protein than the group of sinus rhythm both in left atrium and right atrium with significant difference. Compared with sinus rhythm group, ratio of L-type $\mathrm{Ca}^{2+} / \mathrm{GAPDH}$ protein and SR $\mathrm{Ca}^{2+}$-ATPase/GAPDH protein in paroxysmal AF group decreased with no statistical significance. 3. Level of L-type $\mathrm{Ca}^{2+}$ and SR $\mathrm{Ca}^{2+}$-ATPase mRNA of the chronic AF group declined than sinus rhythm group both in left and right atrium with statistical differences.

Conclusion L-type calcium channel and SR $\mathrm{Ca}^{2+}$-ATPase mRNA and the amount of protein changed significantly in the patients with chronic AF and not significantly in paroxysmal AF group. There was statistical significance between left and right atrium.

\section{e0357 DIAGNOSTIC VALUE ADENOSINE STRESS ${ }^{\text {99M }}$ TC-MIBI GATED MYOCARDIAL PERFUSION IMAGING FOR CORONARY ARTERY DISEASE}

doi:10.1136/hrt.2010.208967.357

${ }^{1}$ Wang Lijuan, 'Li Xiaojuan, ${ }^{1}$ Sun Yingxian, ${ }^{2} \mathrm{Li} \mathrm{Na},{ }^{2} \mathrm{Li}$ Yaming. ${ }^{1}$ Department of Cardiology The First Affiliated Hospital of China Medical University Shenyang, China; ${ }^{2}$ Department of Nuclear Medicine The First Affiliated Hospital of China Medical University Shenyang, China

Objective Stress ${ }^{99 \mathrm{~m}}$ Tc-MIBI myocardial perfusion imaging (MPI) be useful in evaluating myocardial ischaemia and judging coronary artery stenosis. The purpose of this study was to evaluate the sensitivity, specificity and accuracy of adenosine stress ${ }^{99 \mathrm{~m}} \mathrm{Tc}-\mathrm{MIBI}$ gated myocardial perfusion SPECT (G-MPI) for the diagnosis of coronary artery disease (CAD).
Materials and methods The subjects were 46 patients diagnosed or suspected $\mathrm{CAD}$, including forty with angina, six with old myocardial infarction. Adenosine stress G-MPI were performed 90 min after injection of adenosine, and resting G-MPI performed in same day. All patients underwent coronary artery angiography within two weeks. Significant stenosis was defined when the coronary artery intraluminal stenosis $\geq 50 \%$. Analyse the imaging and calculate the sensitivity, specificity and accuracy of adenosine stress G-MPI in diagnosing $\mathrm{CAD}$ and judging coronary artery stenosis. Do correlation analysis of left ventricular systolic function (left ventricular ejection fraction) between adenosine stress ${ }^{99 \mathrm{~m}} \mathrm{Tc}-\mathrm{MIBI}$ G-MPI and echocardiography.

Results The sensitivity, specificity, accuracy, positive predictive value and negative predictive value of adenosine stress ${ }^{99 \mathrm{~m}} \mathrm{Tc}-\mathrm{MIBI}$ G-MPI for the diagnosis of CAD were $87.0 \%, 85.7 \%, 86.6 \%, 93.8 \%$, and $72.7 \%$ respectively. The sensitivity, specificity and accuracy for the diagnosis of LAD, LCX and RCA stenosis were $88.2 \%, 90.9 \%$, $88.9 \% ; 78.6 \%, 83.3 \%, 80.0 \%$; $90.5 \%, 81.8 \%, 87.5 \%$ respectively. LVEF-G-MPI correlated with LVEF-UCG significantly, with a correlation coefficient of 0.885 ( $R=0.0001)$.

Conclusions In Conclusion, stress adenosine ${ }^{99 m}$ Tc-MIBI G-MPI have provided better sensitivity, specificity and accuracy in the diagnosis of $\mathrm{CAD}$, and is probably an accurate method for detecting coronary artery stenosis. It can be used in evaluating left ventricular function, especially for patients unsuited in the exercise MPI

\section{e0358 PROFIBROTIC INFLUENCE OF HIGH GLUCOSE ON HUMAN CARDIAC FIBROBLAST FUNCTIONS}

doi:10.1136/hrt.2010.208967.358

${ }^{1}$ Jufang Chi, ${ }^{1}$ Hangyuan Guo, ${ }^{1}$ Fang Peng, ${ }^{1}$ Biao Yang, 'Longbin Liu, ${ }^{2}$ Hiroyasu Uzui. ${ }^{1}$ Department of cardiology, Shaoxing people's hospital; ${ }^{2}$ Department of cardiology, Faculty of Medical Sciences

Aims Recent studies have demonstrated an important role of chronic high glucose concentration for collagen deposition in fibroblasts. However, little is known about the action of angiotensin II type 1 recepter blocker and inflammatory cytokines on ACE inhibitor on matrix metalloproteinase (MMP) regulation and collagen synthesis in human cardiac fibroblasts. In this article, we determined the influence of chronic high glucose concentration on human cardiac fibroblasts functions and the effects of $606 \mathrm{~A}$ and imidaprilat in these responses.

Methods and results Human cardiac fibroblasts were long-time exposure in normal or high glucose media in the absence or presence of $606 \mathrm{~A}$ or imidaprilat. We have determined their MMP-2 activities by using in-gel zymography. In addition, the collagen IV synthesis was evaluated by the means of ELISA. Results show that chronic high glucose concentration inhibits the activity of MMP-2 and accelerates collagen IV synthesis. When Equimolar mannitol was used as an osmotic control, the activity inhidition of MMP-2 were also observed, however, it is not as strong as that by using high glucose. Inhibition of MMP-2 activity and enhancement of collagen IV synthesis were reserved incompletely by 606A. But complete reservation of MMP-2 activity and collagen IV synthesis was observed by using imidaprilat in cultured media in the experiments.

Conclusions Chronic high glucose inhibits the activity of MMP-2 and increases collagen IV synthesis by means of regulating MMP-2 mRNA expression in human cardiac fibroblasts through osmotic and non-osmotic pathways. Inhibition of MMP-2 activity and enhancement of collagen IV synthesis were reserved incompletely by 606A. But complete reservation of MMP-2 activity and collagen IV synthesis was observed by using imidaprilat in cultured media in the experiments. 